\title{
Numerical Study of the Routes toward Chaos of Natural Convection within an Inclined Enclosure
}

\author{
P. F. Kieno ${ }^{1 *}$, A. Ouédraogo ${ }^{1}$, O. M. Zongo ${ }^{2}$, J. D. Bathiébo ${ }^{1}$, and B. Zeghmati ${ }^{3}$ \\ ${ }^{1}$ Laboratoire d'Energies Thermiques Renouvelables, Département de physique, UFR Sciences Exactes \\ et Appliquées, Université de Ouagadougou, 03 BP 7021 Ouagadougou 03, Burkina Faso \\ ${ }^{2}$ Laboratoire de Mahtématiques Appliquées, Département de physique, UFR Sciences Exactes et \\ Appliquées, Université de Ouagadougou, 03 BP 7021 Ouagadougou 03, Burkina Faso \\ ${ }^{3}$ Laboratoire de Mahtématiques et Physiques des Systèmes (MEPS), Université de Perpignan, 52, \\ avenue Paul Alduy 66860 Perpignan Cedex, France
}

Received 22 May 2012, accepted in final revised form 25 November 2012

\begin{abstract}
Two-dimensional numerical study of transient natural convection in an inclined cubic cavity filled with air using stream function-vorticity form for the Navier-Stokes equations has been carried out to explore the route toward chaos. The hot and cold vertical walls are maintained isothermal at temperature $T_{c}$ and $T_{h}$ respectively and the other walls are adiabatic. Two angles of inclination of the cavity $25^{\circ}$ and $65^{\circ}$ are considered. Transfers equations are solved using finite-difference discretization procedures. The study predicts various critical Rayleigh numbers for the two tilted angles characterizing the variation of the attractor behaviour and shows that the larger the Rayleigh number is, the more sensitive the attractor becomes to time step and meshes size. The routes toward the chaos followed by the attractor are: limit point / limit cycle / $T^{2}$ torus / cycle fitted on a $T^{2}$ torus / chaos / $T^{2}$ torus / cycle fitted on a $T^{2}$ torus / chaos when the Rayleigh number increases. The analysis confirms also the bifurcation of the attractor from a limit point to a limit cycle via an overcritical Hopf bifurcation for a Rayleigh number between $1.95 \times 10^{6}$ and $1.96 \times 10^{6}$.
\end{abstract}

Keyswords: Natural convection; Limit point; Limit cycle; $T^{2}$ torus; Poincaré section; Lyapunov exponent; Chaos.

@ 2013 JSR Publications. ISSN: 2070-0237 (Print); 2070-0245 (Online). All rights reserved.

doi: http://dx.doi.org/10.3329/jsr.v5i1.10709 $\quad$ J. Sci. Res. 5 (1), 105-117 (2013)

\section{Introduction}

Natural convection heat transfer under different physical configurations has been studied extensively numerically and experimentally due to its wide applications such as thermal storage plants, thermal buildings [1-2], industrial processes such cooling of electronic fitting [3], stochastic climate dynamics [4]. The spatio-temporal behaviour of fluid motion in cavities with heated and cooling vertical walls and horizontals walls adiabatic is

\footnotetext{
*Corresponding author: fkieno@univ-ouaga.bf
} 
qualitatively different depending on aspect ratio values and the Rayleigh number [5-6]. Generally, when Rayleigh number is small, the flow is laminar and becomes more complicated [7] as Rayleigh number increases. In Rayleigh-Bénard convection, the flow shows, when the aspect ratio is small, a variety of routes to chaos such as quasi-periodic and intermittencies, depending on the Prandtl number [8]. Spatio-temporal intermittencies appear with large aspect ratio [9-11]. Yang [12] showed that more new and refined experimental and numerical techniques are needed to get better and more accurate resolutions of the spatial and temporal flow details. Four routes to chaos are frequently observed in low-dimensional chaotic systems: the quasi-periodic route via a sequence of Hopf bifurcations, the period-doubling cascade route [13-14], the direct transition to chaos via intermittency [15], and the homoclinic catastrophe. A numerical study of the bifurcation structure and stability of multiple solutions for laminar mixed convection in a rotating curved duct of square cross-section showed that flow and temperature fields on various solution branches are symmetric/asymmetric multi-cells patterns [16]. Furthermore, stable steady 2-D solution, stable multi-cell solution, periodic oscillation, chaotic oscillation and symmetric -breaking oscillation led by sub harmonic bifurcation are displayed and the sub harmonic bifurcation is identified to be another route to the chaos.

A numerical simulation [17] of the two-dimensional thermal convection in a rectangular cavity showed that the pitchfork bifurcation is structurally unstable to the inclination angle of the cavity if the bifurcation flow is anti-symmetric, and is structurally stable if it is symmetric in the horizontal direction. The study of convection heat transfer in a square enclosure, with $45^{\circ}$ angle inclination [18-19], showed that the larger the Rayleigh number is, the more sensitive the attractor becomes to time steps and mesh grids. The system transits from a fixed point toward chaos via a limit cycle, a period doubling cascade, periodic windows, and tangential bifurcation.

In this research, natural convection heat transfer in a cavity inclined with respect to the horizontal plane is studied numerically. The cavity is filled with air. The effect of the Raleigh number and the inclination angle of the cavity on flow and heat transfer and the route toward chaos are investigated for with aspect ratio equal to1.

\section{Formulation of the Problem}

A schematic representation of the system under study is shown in Fig. 1.

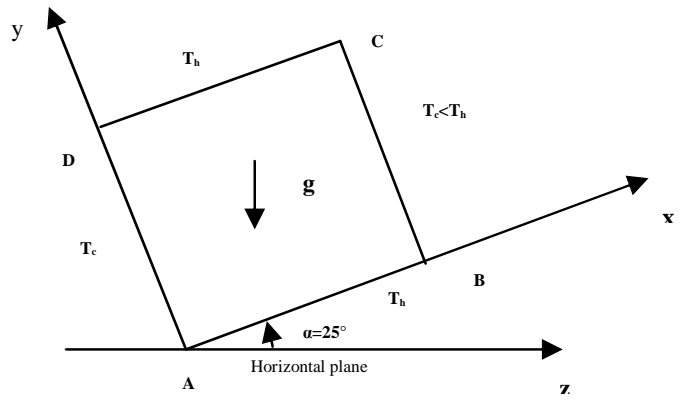

Fig. 1. Physical layout of the system. 
We consider the unsteady natural convection heat transfer in a cubic cavity filled with air inclined with respect to the horizontal plane (Fig.1). Initially, the fluid (air) in the cavity is at rest at a temperature $T_{c}$ and at time $\mathrm{t}_{0}$, the vertical sidewalls (AD and CD) are heated to $T_{c}$ while the sidewalls $\mathrm{BC}$ and $\mathrm{AD}$ are maintained at temperature $T_{h}$; so that $T_{h}>T_{c}$. It is assumed that flow and heat transfer are two-dimensional. The flow is described by NavierStokes and energy equations with the Boussinesq assumption [20]. Navier-Stokes equation, transformed using the stream function formulation and the energy equation are written in non dimensional form in a Cartesian coordinate system:

$$
\begin{aligned}
& \frac{\partial T}{\partial t}+\frac{\partial(u T)}{\partial x}+\frac{\partial(v T)}{\partial y}=\frac{\partial^{2} T}{\partial x^{2}}+\frac{\partial^{2} T}{\partial y^{2}} \\
& \frac{\partial \omega}{\partial t}+\frac{\partial(u \omega)}{\partial x}+\frac{\partial(v \omega)}{\partial y}=R a \cdot \operatorname{Pr}\left[\cos \alpha \frac{\partial T}{\partial x}-\sin \alpha \frac{\partial T}{\partial y}\right]+\operatorname{Pr}\left[\frac{\partial^{2} \omega}{\partial x^{2}}+\frac{\partial^{2} \omega}{\partial y^{2}}\right] \\
& \frac{\partial^{2} \psi}{\partial x^{2}}+\frac{\partial^{2} \psi}{\partial y^{2}}=-\omega
\end{aligned}
$$

with: $\left[x=x^{*} / H\right],\left[y=y^{*} / H\right], T=\frac{T^{*}-T_{f r}^{*}}{T_{c h}^{*}-T_{f r}^{*}}, \omega=\omega^{*} \cdot H^{2} / a, \psi=\psi^{*} / a$ and

$t=t * a / H^{2}$

where $\mathrm{T}$ represents the dimensionless temperature, $\omega$, the vorticity and $\psi$ the stream function. $R a$ is Rayleigh number and $\operatorname{Pr}$ Prandtl number. The associated initial and boundary conditions are:

-Initial conditions

Natural convection in the cavity is produced by temperature difference between hot and cold vertical walls. Initially, the cavity walls are supposed to be of the same temperature. Therefore, we used standard conditions

- $\forall t<t_{0} ; u=0 ; v=0 ; \psi=0 ; T=0$

where $t_{0}$ is the starting (initial) time.

-Boundary conditions

$\forall t \geq t_{0}$

- For $0 \leq x \leq 1 ; y=0$ and $y=1$

$u=0 ; v=0 ; \psi=0$ and $T=1$

- For $0<y<1 ; x=0$ and $x=1$

$u=0 ; v=0 ; \psi=0$ and $T=0$ 
In practical applications, a quantity of physical interest is to be determined, which is heat transfer at the walls. This may be obtained in term of local $N u$ sselt number, $N u_{A B}(x), N u_{D C}(x)$ respectively for heated sides walls and $N u_{A D}(y), N u_{B C}(y)$ respectively for cooled sides walls, from the following relations:

$$
\begin{aligned}
& N u_{A B}(x)=\left[\frac{\partial T}{\partial y}\right]_{y=0} \text { on the hot wall AB } \\
& N u_{D C}(x)=\left[\frac{\partial T}{\partial y}\right]_{y=1} \text { on the hot wall DC } \\
& N u_{A D}(y)=\left[-\frac{\partial T}{\partial x}\right]_{x=0} \text { on the cooled wall AD } \\
& N u_{B C}(y)=\left[-\frac{\partial T}{\partial x}\right]_{x=1} \text { on the cooled wall BC }
\end{aligned}
$$

The average Nusselt numbers $\overline{N u_{A B}}, \overline{N u_{D C}}$ on the hot walls $\mathrm{AB}, \mathrm{DC}$ respectively and $\overline{N u_{A D}}, \overline{N u_{B C}}$ on the cooled walls $\mathrm{AD}, \mathrm{BC}$ respectively are obtained by integration of the local Nusselt number through the corresponding surface and are given by

$$
\begin{gathered}
\overline{N u_{A B}}=\int_{0}^{1} N u_{A B}(x) d x ; \overline{N u_{D C}}=\int_{0}^{1} N u_{D C}(x) d x \\
\overline{N u_{A D}}=\int_{0}^{1} N u_{A D}(y) d y ; \overline{N u_{B C}}=\int_{0}^{1} N u_{B C}(y) d y
\end{gathered}
$$

The overall Nusselt numbers are defined by:

$$
\begin{aligned}
& N u_{H}=\overline{N u_{A B}}+\overline{N u_{D C}} \text { on the hot walls } \\
& N u_{c}=\overline{N u_{A D}}+\overline{N u_{B C}} \text { on the cooled walls }
\end{aligned}
$$

\section{Numerical Procedure and Validation}

\section{1. Numerical procedure}

Governing Eqs. (1-6) are solved using the Alternating Direction Implicit Method (ADI) in a uniform grid and the Gauss elimination method [18]. The convective terms are discretised by a centred scheme and the wall vorticity boundary condition is determined by Woods correlation [19]. Eq. (3) is solved using a successive over relaxation method [18, 21]. For each time steps, convergence was declared when the following criterion is met.

$$
\frac{\sum_{i} \sum_{j}\left|F^{n+l}(i, j)-F^{n}(i, j)\right|}{\sum_{i} \sum_{j}\left|F^{n+l}(i, j)\right|}<\varepsilon
$$


where $F$ denote $T, \Psi$ or $\omega . \varepsilon=10^{-6}$ for $\Psi$ and $\varepsilon=10^{-5}$ for $T$ and $\omega$. Non-stationary attractor frequencies are determined by the Fast Fourier transform [24-25].

\subsection{Validation}

In order to validate our numerical method, we propose to compare ours results with the most closely related numerical solution [18-26]. We notice that for a $45^{\circ}$ inclined square enclosure, with respect to the horizontal plane and heated from two opposite sides, ours results about the Nusselt numbers and the maximum stream function value align with the results by Skouta et al. [18] (Table 1).

Table 1. Results obtained by Skouta [18] and present calculations.

\begin{tabular}{cccc}
\hline Variables & Skouta [26] & Present calculation & Relative Incertitudes \\
\hline$N u_{c h}$ & 13,305 & 13,308 & $0.02 \%$ \\
$N u_{f r}$ & 13,2988 & 13,309 & $0.08 \%$ \\
$\psi_{\max }$ & 11,289 & 11,210 & $0.7 \%$ \\
\hline
\end{tabular}

To ensure that the results from the numerical study are independent from the computational grid, a grid sensitivity analysis was carried out for several Rayleigh numbers. Computations are carried out with several mesh sizes, several step time and Rayleigh number values [27].

\section{Results and Discussion}

Results are in various forms, including $T^{2}$ torus, strange attractor, Poincaré section and Fourier frequency spectrum for two inclination angles: $25^{\circ}$ and $65^{\circ}$ and Rayleigh number varying between $10^{6}$ and $7.5 \times 10^{6}$.

\subsection{Bifurcation limits point/limit cycle}

For Rayleigh numbers inferior or equal to $1.95 \times 10^{6}$, dynamic variables evolve as time increases toward an asymptotic limit of a steady state. For example, Fig. 2 exhibits for $R a=1.95 \times 10^{6}$ the evolution during the time of the $T m$ (Fig. 2a) and the trajectory in the plane phase (Tm, Psimax) (Fig. 2b). We notice that the system evolves toward an asymptotic limit of a steady state for both inclination angle $\left(25^{\circ}\right.$ and $\left.65^{\circ}\right)$ used in this study. Results on the evolution at fixed point of the Nusselt average number as the Rayleigh number increases can be presented for the inclination angle of $25^{\circ}$ by:

$$
\overline{N u}=1.7178 \times R a^{0.1818}
$$


The gaps between Nusselt average numbers deduced from this correlation and results obtained from the resolution of transfers' equation are less than $1 \%$.

(a)

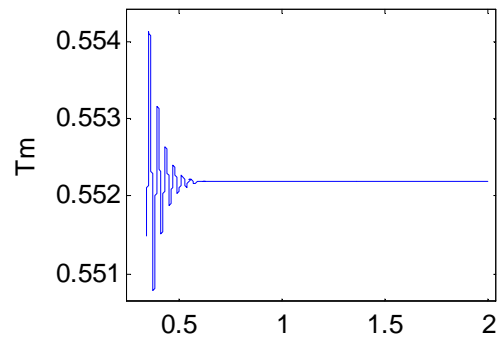

(b)

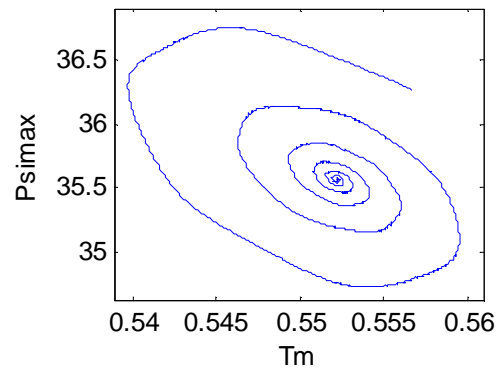

Fig. 2. Representation of the limit point attractor before limit cycle for $R a=1,95 \cdot 10^{6}$. a): Temporal signal of the temperature $T m, \mathrm{~b}$ ): Trajectory in the phase plane (Tm, Psimax).

(a)

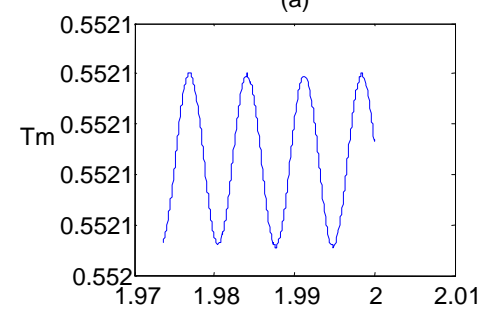

(c)

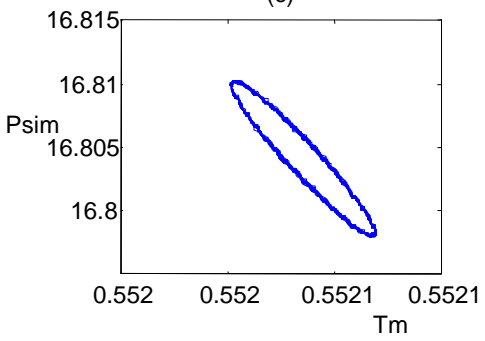

(b)

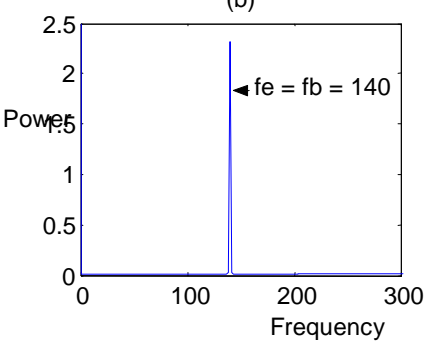

$10^{-6} . \Delta t$

$10^{-6}$

Fig. 3. Reprsentation of the limit cycle attrator for $R a=1,96 \cdot 10^{6}$,. a): Temporal signal of $T \mathrm{~m}$ (b): Amplitude spectrum of $T m,(\mathrm{c})$ : Trajectory in the (Tm, Psim) phase plane.

As the Rayleigh number increases, the flow intensity and the computing time to obtain the attractor increase. We report on Fig. 6 the evolution during time of Tm (Fig. 3a) and the trajectory in plane phase (Tm, Psim) (Fig. 3b). We get the value of this number for which the attractor becomes periodic as the Rayleigh number increases. This critical Rayleigh number is between $1.95 \times 10^{6}$ and $1.96 \times 10^{6}$ (Fig. 3). The plotting of the amplitude spectrum of dynamic variables obtained by Fast Fourier Transform corroborates the existence of a limit cycle (Fig. 3c). 
We notice that with $R a=1.958 \times 10^{6}$ around the bifurcation point, the frequency is constant (Fig. 4b) whereas the amplitudes increase linearly with $\left(R a-1.958 \times 10^{6}\right)^{1 / 2}$ (Fig. 4b), meaning that the bifurcation from the point attractor to the limit cycle is an overcritical Hopf bifurcation [21].

(a)
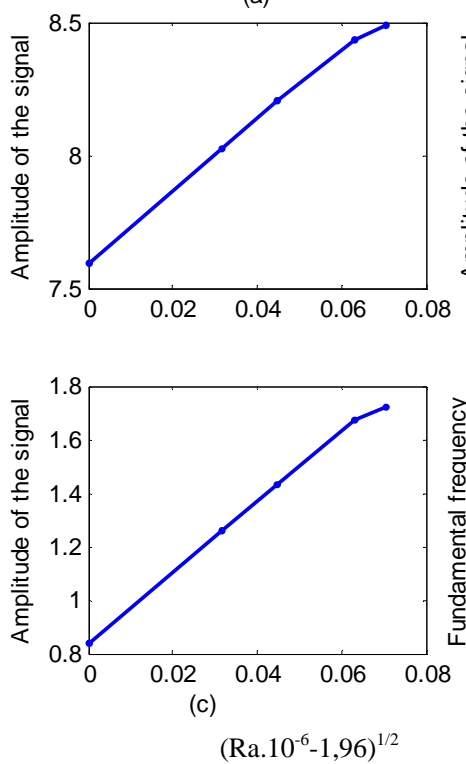

(b)
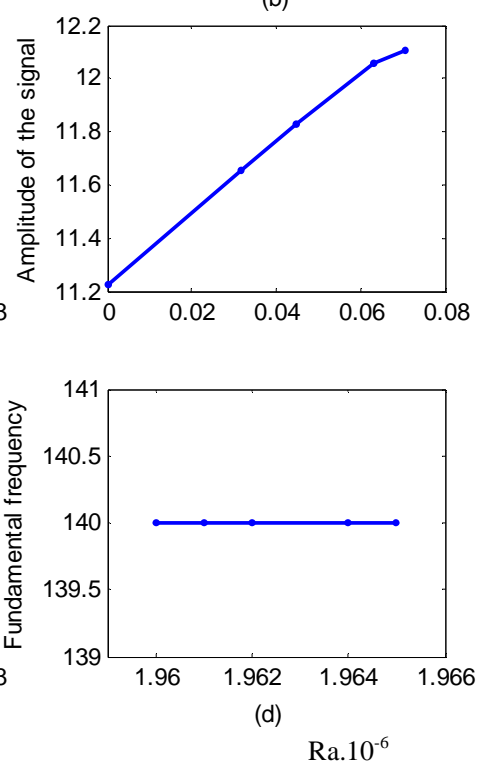

Fig. 4. Influence of the Rayleigh number around the bifurcation point for angle of $25^{\circ}$. a) Variations of Nuch amplitude versus $\left(\text { Ra. } 10^{-6}-1,960\right)^{1 / 2}$, (b) Variations of Psim amplitude versus $\left(\operatorname{Ra} .10^{-6}-1,960\right)^{1 / 2}$, (c) Variations of $T m$ amplitude versus $\left(\operatorname{Ra} \cdot 10^{-6}-1,960\right)^{1 / 2}$, (d) Variations of spectrum fundamental frequency amplitude of Nuch, Psim and Tm versus $R a \cdot 10^{-6}$.

\section{2. $T^{2}$ torus}

Fig. 8 shows two frequencies whose irrational ratio $R a \approx 3.5 \times 10^{6}$. This implies a second value of critical Rayleigh number around $3.5 \times 10^{6}$, for which the periodic regime undergoes a transition by destruction of the linearly stability toward a periodic cycle and $T^{2}$ torus. We notice for the two inclination angles, the stability of the quasi-periodicity of the two frequencies for Rayleigh numbers, varying between $3.5 \times 10^{6}$ and $3.64 \times 10^{6}$. For example, Fig. 8 gives $R a=3.613 \times 10^{6}$, the temporal signal of $N u_{h}$ (Fig. 5a), the spectrum amplitude of $N u_{h}$ (Fig. 5b), the trajectory in the plane phase (Fig. 5c) and the Poincaré section (Psimin, Psimax; Tm = 0.54) (Fig. 5d) which includes two heaps of points. The power spectra of Nuh, Tm, Psim and Psimax shows the presence of two new rays and the harmonics of which are a linearly combination put as $m_{1} f 1+m_{2} f 2$ in which $\mathrm{m}_{1}$ and $\mathrm{m}_{2}$ are natural integers. 


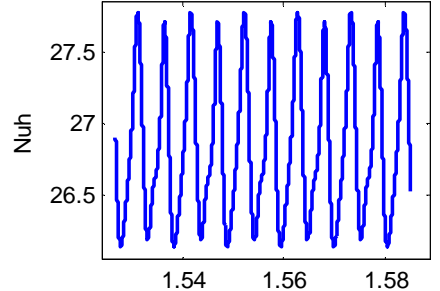

(a)

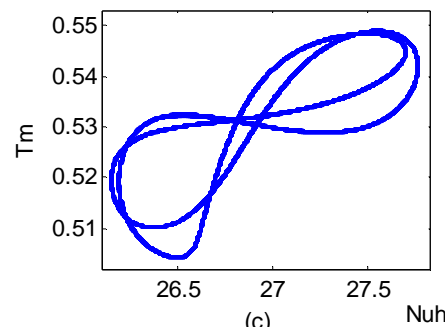

(c)

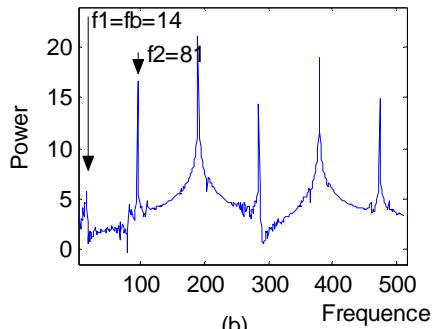

(b)

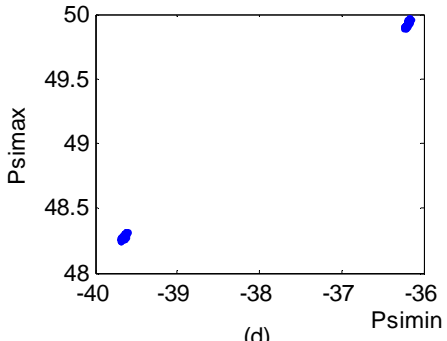

Fig. 5. Illustration of the quasi-periodic state for $R a=3,613.10^{6}$. (a): temporal signal of $N u h$, (b) Power spectra of $N u h$, (c) Trajectory in the $(N u h, T m)$ plane, (d) Poincaré section (Psimin, psimax) for $T m=0,54$

\subsection{Cycle fitted on a $T^{2}$ torus}

We report on Fig. 6 the evolution, for $R a=3.7 \times 10^{6}$, during $N u_{h}$ time (Fig. 6a), its power spectrum (Fig. 6b), the trajectory in the plane phase $\left(N u_{h}, \mathrm{Tm}\right)$ (Fig. 6c) and the Poincare section (Psimin, Psimax; $T m=0.56$ ) (Fig. 6d). We notice that ratio fe/fb is rational for Rayleigh numbers near $3.65 \times 10^{6}$ and Fourier Spectrum rays are harmonics of the lower frequency. So, both frequencies hook and the flow periodic are characterised by a $T^{2}$ torus.

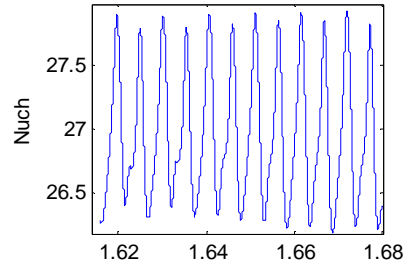

(a)

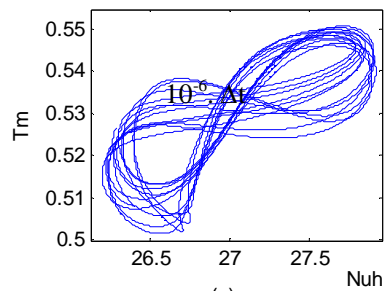

(c)
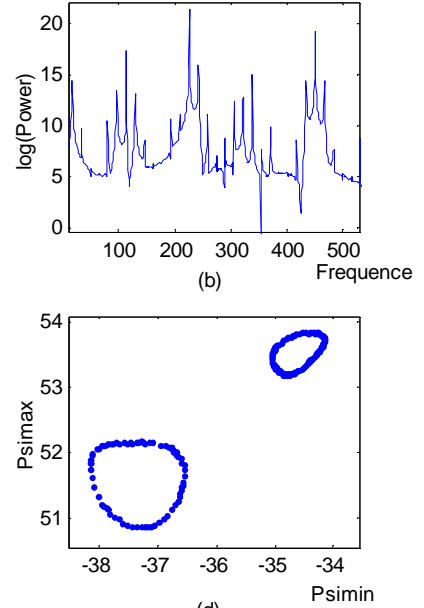

(d)

Fig. 6. Illustration of a cycle fitted on a $T^{2}$ torus for $R a=3,7 \times 10^{6}$. (a) Temporal signal of $N u h$ (b) Power spectra of $N u h$, (c) Trajectory in the $(N u h, T m$ ) plane, (d) Poincaré section (Psimin, Psimax) for $T m=0.54$. 


\subsection{Destabilization of the system by appearance of a chaotic window}

Increasing $R a$ from $4.10 \times 10^{6}$ to $4.68 \times 10^{6}$ leads to the onset of peaks for which the power spectrum increases to become dense and in chaotic state (Fig. 7). The chaotic state seems to be confirmed by the Poincaré section and by the positive Lyapanov exponent (Table 6). This result aligns with those by Skouta [18].

Lets us now examine the impact of the increase of Rayleigh number on the natural convection flow. We notice that with $R a=4.7 \times 10^{6}$ and $25^{\circ}$ inclination angle, both frequencies are as follow: $f l=47$ and $f 2=53$, for which the ratio is irrational (Fig. 8). It means the apparition of $T^{2}$ torus. The $T^{2}$ torus is observed until $R a=5.6 \times 10^{6}$ for $25^{\circ}$ inclination angle. The increasing in the inclination angle leads to an increase in the range of values of the Rayleigh number for which $T^{2}$ torus exists. We notice that for an inclination angle of $65^{\circ}, T^{2}$ Torus appears until $R a=5.00 \times 10^{6}$.

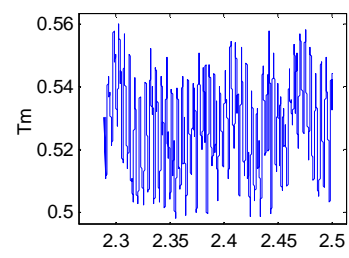

(a)

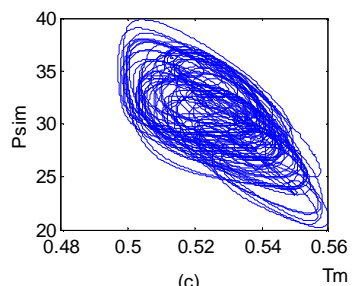

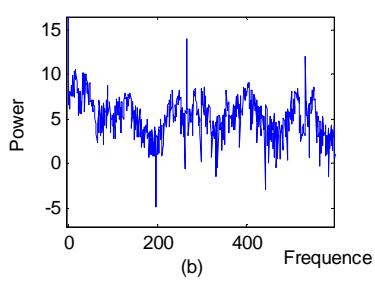

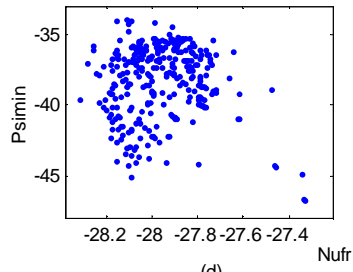

(d)

Fig. 7. Illustration of the chaotic state flow for $R a=4,4 \cdot 10^{6}$. (a): Temporal signal of $T m$ (b): Power spectra of Tm, (c) Trajectory in the (Tm, Psim) plane, (d): Poincare section (Nufr, Psimin) for Tm = 0.52 .

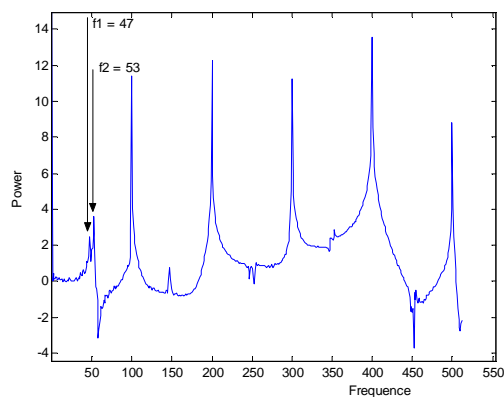

Fig. 8. Power spectra of $T m$ for $R a=4,7 \cdot 10^{6}$ when $\alpha=25^{\circ}$. 


\subsection{Strange attractor}

For the both inclination angles, the attractor is, for $R a=5.7 \times 10^{6}$ or $R a=5.5 \times 10^{6}$, a cycle fitted on $T^{2}$ torus. We notice that for an inclination angle of $25^{\circ}$ and $R a=5.9 \times 10^{6}, R a=$ $6 \times 10^{6}$ the $T m$ temporal signal, its power spectrum and its great sensitive dependence on initial conditions as the trajectory in the plane phase (Tm, Psim) are characteristic of a chaotic attractor as illustrated in Fig. 9. However, the Tm temporal signal shows irregular amplitudes characterising the chaos state (Fig.9a). The Tm power spectrum presents a noise (Fig.9b). So, it is very difficult to determine the frequencies. The Poincaré section is formed of scattered points (Fig. 9d). Results reported above show that the attractor is strange. As Rayleigh number continues to increase, the chaotic regime expands.

We calculated the largest Lyapunov exponent values versus $R a$ using the relation below:

$$
N u\left(T_{01}, t\right)-N u\left(T_{02}, t\right)=\left[N u\left(T_{01}, 0\right)-N u\left(T_{02}, 0\right] \exp (L y . t)\right.
$$

where $T_{01}=0$ and $T_{02}=10^{-6}$ are initial temperatures.

The great positive Lyapanov exponent increasing with Rayleigh numbers (Table 2) shows the intensification of the chaos and that the attractor is strange.

Table 2. The largest Lyapunov exponent values for different Rayleigh numbers when tilt angle is $65^{\circ}$.

\begin{tabular}{cc}
\hline Rayleigh number & Largest Lyapunov exponent \\
\hline $6.00 .10^{6}$ & 3.32 \\
$6.20 .10^{6}$ & 3.87 \\
$6.50 .10^{6}$ & 4.41 \\
\hline
\end{tabular}

Fractal dimensions of attractors have been calculated using Saraille code FD3 [28-29] which uses the iterations of physical variables for example Nuch, Psimax, Tm and their successive derivatives with regard to time. This sets sizes, defines a pseudo-space of the phases of dimension $n+1$, and where $n$ is the number of derivatives. When $n$ increases, FD3 gives values which can increase until the limit corresponding to the dimension $\mathrm{d}$ of the attractor with nearly $10 \%$ incertitude margin. Calculations give $d>2$ for a number of Rayleigh above $6.10^{6}$. The use of FD3 confirms the appearance of a strange attractor when $\mathrm{d}$ is above 2 . 


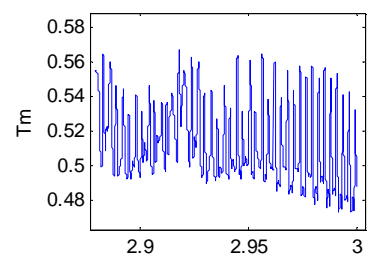

(a)

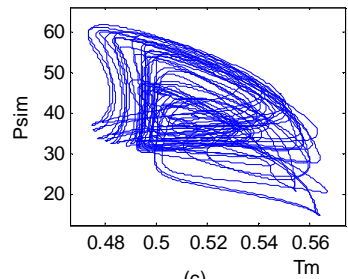

(c)

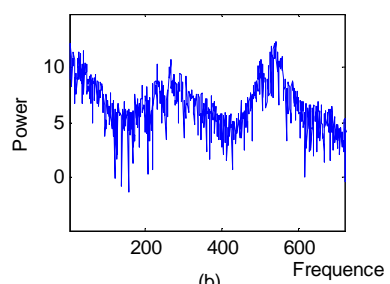

(b)

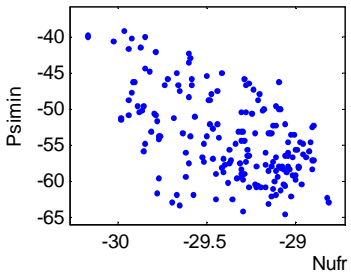

(d)

Fig. 9. Representation of chaotic attractor for $R a=6 \cdot 10^{6}$ and $\boldsymbol{\alpha}=25^{\circ}$. (a): Temporal signal of $T m$, (b): Power spectra of Tm, (c): Trajectory in the (Tm, Psim) plane, (d): Poncaré section (Nufr, Psimin) for $T m=\mathbf{0 . 5 2}$.

\section{Conclusion}

Natural convection in an enclosure inclined to the horizontal plane was numerically investigated in this study in order to determine the routes to chaos. This enclosure is heated from two opposite side and cooled on the other two sides. Transfers equations are solved using the vorticity-stream function formulation, the alternating direction implicit method (ADI) and the Gauss elimination method. The impact of Rayleigh number and the inclination angle are examined in detail. Major results are:

- The routes to the chaos for complementary angles $\left(25^{\circ}\right.$ and $\left.65^{\circ}\right)$ are identical.

- With $25^{\circ}$ inclination angle, the attractor is a fixed point for Rayleigh number less than $1.95 \times 10^{6}$; it transits from fixed point toward limit cycle via an overcritical Hopf bifurcation for a value of Rayleigh number between ranging $1.95 \times 10^{6}$ and $1.96 \times 10^{6}$.

- For the Rayleigh number varying between $5.00 \times 10^{6}$ and $3.64 \times 10^{6}$, the limit cycle undergoes a second Hopf bifurcation which turns it into $T^{2}$ torus. A cycle aligned on $T^{2}$ torus appears after a hooking of frequencies which continues until $R a=4.00 \times 10^{6}$.

- A chaotic sequence seems to appear for Ra varying between $4.10 \times 0^{6}$ to $4.68 \times 10^{6}$ and a laminar flow appears from $R a=4.7 \times 10^{6}$.

- For $R a$ higher than $5.6 \times 10^{6}$, a hooking of frequencies brings back the $T^{2}$ torus to a limit cycle fitted on it.

- When Rayleigh number increases up to $5.9 \times 10^{6}$, the attractor is chaotic again. 


\section{Acknowledgements}

This work was supported by the Coopération Universitaire Institutional, Universités Francophones de Belgique (CIUF) with the financial support from the A.G.C.D. The authors would like to sincerely thank them.

\section{Nomenclature}

\section{Latin symbols}

$a \quad$ thermal diffusivity $\left(\mathrm{m}^{2} \cdot \mathrm{s}^{-1}\right)$

$\Delta t \quad$ dimensionless time step

$g \quad$ gravitational acceleration $\left(\mathrm{m} . \mathrm{s}^{-2}\right)$

$H \quad$ height of the cavity (m)

$n \quad$ iteration number

$N u_{H} \quad$ global Nusselt number on the hot sides AB and CD $\left[\int_{0}^{1}(\partial T / \partial y)^{\mathrm{y}=0 .} \cdot d x+\int_{0}^{1}(\partial T / \partial y){ }_{\mathrm{y}=1} \cdot d x\right]$

$N u_{C} \quad$ global Nusselt number on the cooled sides AD and BC $\left[-\int_{0}^{1}(\partial T / \partial x)^{\mathrm{x}=0 . \mathrm{dy}}-\int_{0}^{1}(\partial T / \partial x)^{\mathrm{x}=1} \cdot \mathrm{dy}\right]$

$\overline{\mathrm{Nu}}$ mean Nusselt number

$N x \quad$ node number in the $[\mathrm{Ax})$ direction

Ny node number in the [Ay) direction

$\mathrm{Pr} \quad$ Prandtl number: $v / \mathrm{a}$

$R a \quad$ Rayleigh number: $\mathrm{g} \beta\left(T_{h}^{*}-T_{c}^{*}\right) \cdot H^{3} /(v \cdot \mathrm{a})$

Rac critical Rayleigh number

$t \quad$ dimensionless time : $t^{*} a / H^{2}$

$T$ dimensionless temperature

$$
\frac{T^{*}-T_{f r}^{*}}{T_{c h}^{*}-T_{f r}^{*}}
$$

$u \quad$ dimensionless velocity component along $x$ direction : $u^{*} \cdot a / H$

$v \quad$ dimensionless velocity component along $y$ direction $: v^{*} \cdot a / H$

$x, y \quad$ velocity coordinates: $x^{*} / H, y^{*} / H$

\section{Greek symbols}

$\alpha \quad$ inclination angle of the side wall $\mathrm{AB}$ with the horizontal plane (rad).

$\beta \quad$ thermal expansion coefficient $\left(\mathrm{K}^{-1}\right)$

$\lambda \quad$ thermal conductivity $\left(\mathrm{W} \cdot \mathrm{m}^{-1} \cdot \mathrm{K}^{-1}\right)$

$v \quad$ kinematic viscosity $\left(\mathrm{m}^{2} \cdot \mathrm{s}^{-1}\right)$.

$\psi \quad$ dimensionless stream function : $\left(\psi^{*} / a\right)$.

$\omega$ dimensionless vorticity $\left(\omega^{*} \cdot H^{2} / a\right)$.

Subscripts:

c cold surface.

$h$ hot surface.

$m$ middle

$\max$ maximum.

min minimum

$0 \quad$ initial value

Super scripts

* dimensional quantity 


\section{References}

1. C. Inard, P. Depecke, and J-J. Roux, Revue Générale de Thermique 36, 113 (1997). http://dx.doi.org/10.1016/S0035-3159(99)80056-7

2. R. Braconnier, Revue Générale de Thermique Fr. no. 385, 12 (1994).

3. E. K. Lakhal, M. Hasnaoui, P. Vasseur, and E. Bilgen, Revue Générale de thermique no. 392393, 480 (1994).

4. M. D. Chekroun, E. Simonnet, and M. Ghil. Physica D 240, 1685 (2011). http://dx.doi.org/10.1016/j.physd.2011.06.005

5. A. G. Churbanov, Int. J. Heat Mass Transfer 37 (18), 2969 (1994). http://dx.doi.org/10.1016/0017-9310(94)90351-4

6. D. Mukutmoni and K. T. Yang, Int. J. Heat Mass transfer 35 (9) 2145 (1992). http://dx.doi.org/10.1016/0017-9310(92)90059-2

7. T. Yanagita and K. Kaneko, Physica D 82 (3) 288 (1995). http://dx.doi.org/10.1016/0167-2789(94)00233-G

8. D. Mukutmoni and K. T. Yang, J. Heat Transfer 115, 360 (1993). http://dx.doi.org/10.1115/1.2910687

9. J. P. Gollub and S. V. Benson, J. Fluid. Mech. 100 (3), 449 (1980). http://dx.doi.org/10.1017/S0022112080001243

10. K. T. Yang, J. Heat Transfer 110, 1191 (1988). http://dx.doi.org/10.1115/1.3250620

11. D. Mukutmoni and K. T. Yang, Int. J. Heat Mass Transfer 1, 113 (1995). http://dx.doi.org/10.1016/0017-9310(94)00124-E

12. D. Ruelle and F. Takens, Commu. Math. Phys. 20, 167 (1971). http://dx.doi.org/10.1007/BF01646553

13. M. J. Feigenbaum, J. Stat. Phys. 19, 25 (1978). http://dx.doi.org/10.1007/BF01020332

14. D. Mukutmoni and K. T. Yang, J. Heat Transfer 115, 367 (1993). http://dx.doi.org/10.1115/1.2910688

15. J. Guckenheimer and P. Holmes, Non linear Oscillations, Dynamical Systems and Bifurcations of vector fields (Springer-Verlag, New York, 1983).

16. K. T. Yang and L. Wang L, Int. J. Heat Mass Transfer 46, 613 (2003). http://dx.doi.org/10.1016/S0017-9310(02)00329-0

17. J. Muzushima and T. Adachi, J. Phys. Soc. Japan 66 (1), 79 (1997). http://dx.doi.org/10.1143/JPSJ.66.79

18. A. Skouta, M. A. Randriazanamparany, and M. Daguenet, Int. J. Thermi. Sci. 40, 352 (2001). http://dx.doi.org/10.1016/S1290-0729(01)01228-5

19. M. A. Randriazanamparany, A. Skouta, and M. Daguenet, Numer. Heat Transfer Part A, 48, 127 (2005). http://dx.doi.org/10.1080/10407780490454386

20. J. V. Boussinesq, Théorie analytique de la chaleur, vol. 2 (Gauthier-Villars, Paris, 1903).

21. L. C. Woods, Aeronau. Quarterly (1953).

22. S. Douamna, M. Hasnaoui, and B. Abourida, Int. J. Therm. Sci. 38, 797 (1999). http://dx.doi.org/10.1016/S1290-0729(99)80035-0

23. P. Bergé, Y. Pomeau, and C, Vidal, L’ordre dans le chaos, vers une approche déterministe de la turbulence (Hermann, Paris, 1984).

24. Kautz and Richard, Chaos: The Science of Predictable Random Motion (Oxford University Press, 2011).

25. G. De Vahl Davis Int. J. Numer. Meth. Fluids 3, 227 (1983). http://dx.doi.org/10.1002/fld.1650030304

26. P. F. Kieno and A. Ouédraogo, Global J. Pure Appl. Sci. 16 (3), 333 (2010).

27. F. Karen and W. Raymond, Comparative fractal dimensions for daytime and nocturnal surface layer turbulence, $11^{\text {th }}$ Symposium on Boundary Layers and Turbulence, American Meteorological Society, 27-31 March, Charlotte, NC (1995).

28. A. Margaris, Comput. Inform. Sci. 5 (4) 25 (2012). 